\title{
An exact solution for a steady, flowline marine ice sheet
}

\author{
Ed BUELER
}

\begin{abstract}
Department of Mathematics and Statistics and Geophysical Institute, University of Alaska Fairbanks, Fairbanks, AK, USA E-mail: elbueler@alaska.edu
\end{abstract}

\begin{abstract}
G. Böðvarsson's 1955 plug-flow solution for an Icelandic glacier problem is shown to be an exact solution to the steady form of the simultaneous stress-balance and mass-continuity equations widely used in numerical models of marine ice sheets. The solution, which has parabolic ice thickness and constant vertically integrated longitudinal stress, solves the steady shallow-shelf approximation with linear sliding on a flat bed. It has an elevation-dependent surface mass-balance rate and, in the interpretation given here, a contrived location-dependent ice hardness distribution. By connecting Böðvarsson's solution to the Van der Veen (1983) solution for floating ice, we construct an exact solution to the 'rapid-sliding' marine ice-sheet problem, continuously across the grounding line. We exploit this exact solution to examine the accuracy of two numerical methods, one grid-free and the other based on a fixed, equally spaced grid.
\end{abstract}

KEYWORDS: glacier modelling, ice dynamics, ice-sheet modelling

\section{INTRODUCTION}

Early theoretical glaciology created two fundamentally different parabolic profiles as the shapes of steady flowline ice sheets lying on flat beds (Fig. 1). One was the profile of an ice sheet with perfect plasticity (E. Orowan comment in BGS, 1949; Nye, 1952) and the other the profile for a sliding 'plug' flow (Böðvarsson, 1955). These global views of free surface flows in glaciology focus on different aspects of the problem and they come to rather different conclusions. Up to scaling, one is of the form $x=1-z^{2}$ (Orowan-Nye) and the other is of the form $z=1-x^{2}$ (Böðvarsson). The former perfect-plasticity solution has a central peak at the highest point of the ice sheet, and a margin with unbounded surface gradient. The latter plug-flow solution has a smooth dome and a finite-slope, wedge-shaped margin.

This paper shows how to combine Böðvarsson's solution with a well-known exact solution for an ice shelf (Van der Veen, 1983, 2013) to generate an exact solution for a flowline, steady marine ice sheet. It is shown in Figure 2. This exact solution simultaneously solves the steady masscontinuity equation and the so-called shallow-shelf approximation (SSA; Weis and others, 1999) stress balance. It is an exact solution in the rapidly sliding marine ice-sheet case (Schoof, 2007), the model addressed by the MISMIP (Marine Ice-Sheet Model Intercomparison Project) intercomparison (Pattyn and others, 2012). After presenting the model equations and constructing the exact solution in the next two sections, we examine the errors from two different numerical methods. We observe, through linearization of the equations around the exact solution, that the grounding line generates a strong 'stiffness' contrast, in the sense of numerical analysis.

\section{CONTINUUM MODEL}

\section{Model equations}

Our model equations describe the steady-state, flat-bed case of the rapid-sliding model of Schoof (2007, eqns (2.1-2.5)), but we restrict our consideration to the linear-sliding case. The primary unknowns in these equations are the ice thickness, $H(x)$, velocity, $u(x)$, and vertically integrated longitudinal stress, $T(x)$ (Schoof, 2006), where $x$ is the flowline distance. Using the notation of Table 1 , the equations are

$$
\begin{gathered}
(u H)_{x}-M=0, \\
T_{x}-\beta u-\rho g H h_{x}=0, \\
T=2 B H\left|u_{x}\right|^{\frac{1}{n}-1} u_{x} .
\end{gathered}
$$

Here the subscript $x$ denotes the derivative. Equations (1) and (2) are the mass-continuity and SSA stress-balance equations, respectively, while Eqn (3) defines $T$. In Eqn (1) the climatic-basal mass-balance rate, $M(x)$ (Cogley and others, 2011), also called the accumulation/ablation rate function, combines the surface mass-balance rate and the rate of basal melt/refreeze. In grounded ice the basal shear stress is linear, so $\tau_{\mathrm{b}}=-\beta u$ (MacAyeal, 1989) appears in Eqn (2), with $\beta=0$ in floating ice, so the same linear form holds throughout. The coefficient $B(x)$ in Eqn (3) is called the ice 'hardness'.

Let $\omega=1-\rho / \rho_{\mathrm{w}}$ be the 'Archimedean factor', which relates ice surface elevation to thickness in floating ice. Let $z_{0}$ be the elevation of the ocean surface. The grounded ice rests on bedrock at elevation $b$, which is a constant in this paper. By the flotation criterion,

$$
h= \begin{cases}H+b, & \rho H \geq \rho_{\mathrm{w}}\left(z_{\mathrm{o}}-b\right) \\ \omega H+z_{\mathrm{o}}, & \rho H<\rho_{\mathrm{w}}\left(z_{\mathrm{o}}-b\right)\end{cases}
$$

defines the ice surface elevation in grounded and floating cases, respectively. Using the same flotation criterion we can clarify the basal shear stress,

$$
\beta= \begin{cases}k \rho g H, & \rho H \geq \rho_{\mathrm{w}}\left(z_{\mathrm{o}}-b\right) \\ 0, & \rho H<\rho_{\mathrm{w}}\left(z_{\mathrm{o}}-b\right)\end{cases}
$$

where we have scaled $\beta=k \rho g H$ with the ice overburden pressure, $\rho g H$, following Böðvarsson (1955).

Equations (1-5) apply on an interval $0<x<x_{\mathrm{C}}$, where $x_{\mathrm{C}}$ is the floating calving front. We must solve for the location, $x=x_{\mathrm{g}}$, of the grounding line, at which we know $\rho H\left(x_{\mathrm{g}}\right)=\rho_{\mathrm{w}}\left(z_{\mathrm{o}}-b\right)$. 


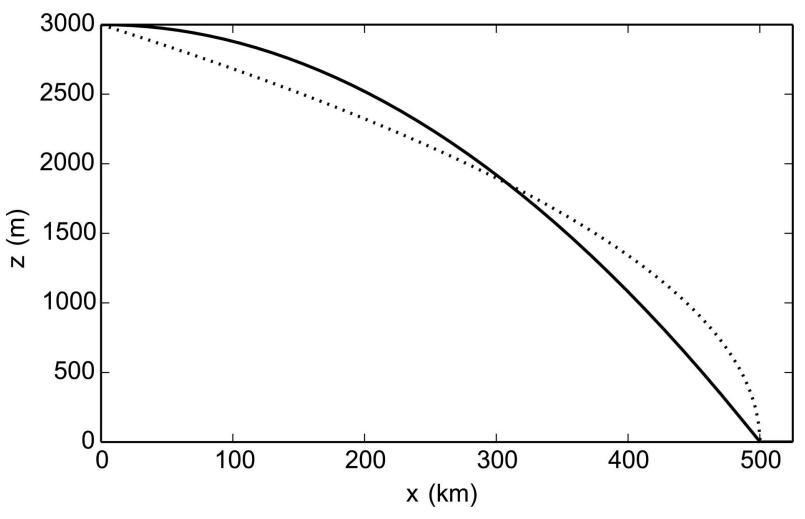

Fig. 1. The parabolas by Orowan (comment in BGS, 1949) and Nye (1952) (dotted curve) and by Böðvarsson (1955; solid curve) for steady, flowline ice sheets on flat beds. A dome thickness of $H_{0}=3000 \mathrm{~m}$ and a length of $L_{0}=500 \mathrm{~km}$ are chosen for concreteness.

For the exact and numerical solutions in this paper, Eqns (1-5) are augmented by boundary conditions:

$$
\begin{gathered}
u(0)=u_{\mathrm{a}}>0, \quad H(0)=H_{\mathrm{a}}>0, \\
T\left(x_{\mathrm{c}}\right)=\frac{1}{2} \omega \rho g H\left(x_{\mathrm{c}}\right)^{2},
\end{gathered}
$$

$$
H, u, T \text { continuous at } x=x_{\mathrm{g}}
$$

where $u_{a}$ and $H_{a}$ simply denote Dirichlet boundary values for velocity and thickness respectively. Here $x=0$ is an upstream location where Dirichlet boundary conditions are applied (Eqn (6)), while at the calving front, $x=x_{C}$, we have the standard hydrostatic pressure imbalance condition, Eqn (7) (Schoof, 2007).

\section{On well-posedness and the grounding line}

Given data $B(x), b, k>0, M(x), x_{c}>0, z_{0}$, along with physical constants $g, n, \rho, \rho_{\mathrm{w}}$, we expect the problem consisting of Eqns (1-8) to be well-posed. To our knowledge

\begin{tabular}{|c|c|c|}
\hline Symbol & Description & Unit or value \\
\hline$B$ & Ice hardness $\left(=A^{-1 / n}\right)$ & $\operatorname{Pas}^{1 / 3}$ \\
\hline$b$ & Bedrock elevation & $\mathrm{m}$ \\
\hline$\beta$ & Sliding coefficient & Pas m ${ }^{-1}$ \\
\hline$g$ & Acceleration of gravity & $9.81 \mathrm{~m} \mathrm{~s}^{-2}$ \\
\hline H & Ice thickness & $\mathrm{m}$ \\
\hline$h$ & Ice surface elevation & $\mathrm{m}$ \\
\hline$k$ & Pressure-scaled sliding coefficient & $s \mathrm{~m}^{-1}$ \\
\hline$M$ & Climatic-basal mass-balance rate & $\mathrm{m} \mathrm{s}^{-1}$ \\
\hline$n$ & Glen exponent in ice-flow law & 3 \\
\hline$\rho$ & Density of ice & $910 \mathrm{~kg} \mathrm{~m}^{-3}$ \\
\hline$\rho_{\mathrm{w}}$ & Density of sea water & $1028 \mathrm{~kg} \mathrm{~m}^{-3}$ \\
\hline$T$ & z-integrated longitudinal stress & Pa m \\
\hline$\tau_{\mathrm{b}}$ & Basal shear stress applied to ice & $\mathrm{Pa}$ \\
\hline$u$ & Horizontal velocity & $\mathrm{ms}^{-1}$ \\
\hline$(x, z)$ & Flowline Cartesian coordinates & $\mathrm{m}$ \\
\hline$x_{\mathrm{g}}$ & Grounding line & $\mathrm{m}$ \\
\hline$x_{\mathrm{C}}^{\circ}$ & Calving front & $\mathrm{m}$ \\
\hline$z_{\mathrm{O}}$ & Ocean surface elevation & $\mathrm{m}$ \\
\hline$\omega$ & Archimedean factor $\left(=1-\rho / \rho_{\mathrm{w}}\right)$ & 0.115 \\
\hline
\end{tabular}
this has not been proved, nor do we attempt to prove it. It is,

Table 1. Notation and SI units or values. Values of physical constants

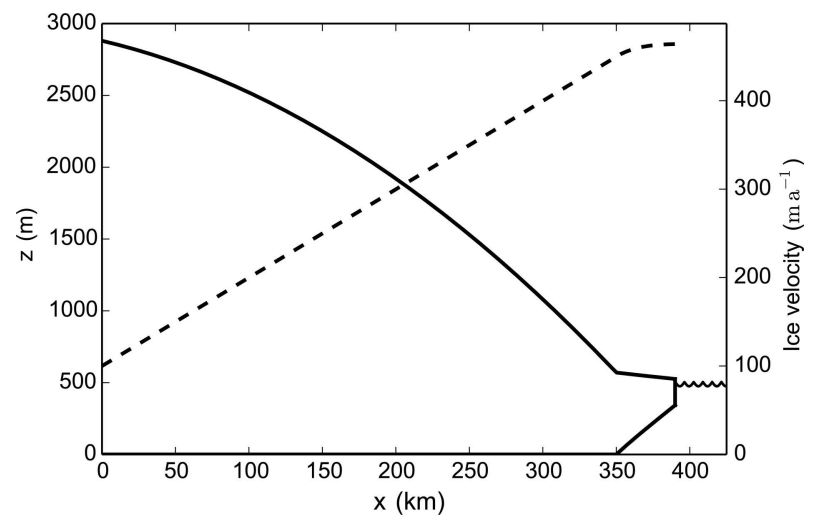

Fig. 2. The geometry (solid curve) and velocity (dashed curve) of an exact solution of the simultaneous steady mass-continuity and SSA stress-balance equations for a marine ice sheet. The solution is Böðvarsson's (1955) when grounded and Van der Veen's (1983, 2013) when floating.

however, now worth considering the smoothness of the solution to Eqns (1-7), including what hypotheses would lead to satisfying Eqn (8) at the free (i.e. unknown) location $x_{\mathrm{g}}$ in the interior of the domain.

Suppose that, for physical reasons, the climatic-basal mass-balance rate, $M(x)$, and ice hardness, $B(x)$, are bounded, and further that $B(x)$ is bounded below by a positive constant. On integrating $M$, Eqn (1) implies that the flux, $q=u H$, is absolutely continuous and bounded. If there is a positive lower bound on thickness, $H$, then we can conclude that the magnitude of $u$ is bounded because $u=q / H$. If the magnitude of the driving stress, $-\rho g H h_{x}$, is bounded then Eqn (2) implies $T$ is absolutely continuous. By Eqn (3) this implies $u$ has a bounded and integrable derivative, and, thus, that $u$ is also absolutely continuous. From these facts we could then return to the flux and write $H=q / u$, which shows $H$ is absolutely continuous away from locations where $u=0$ (e.g. divides). In summary, assuming (i) that an integrable solution $(H, u, T)$ to Eqns (17) exists, (ii) that the functions $M, B$ are bounded and integrable, (iii) that $B$ is bounded below by a positive constant, (iv) that a positive lower bound on thickness exists and $(v)$ that an upper bound on the magnitude of the driving stress exists, then we can regard the conditions of Eqn (8), giving continuity at the grounding line, as properties of the solution instead of as part of the 'imposed' problem statement. Thus, from now on we speak of solving the problem consisting of Eqns (1-7), with the location of the grounding line, $x_{\mathrm{g}}$, unknown, and with Eqn (8) describing proportion of the solution.

\section{EXACT SOLUTION Böðvarsson's parabola}

Böðvarsson (1955) built, based on minimal existing literature, a rigorous theory of the flow of glaciers and ice sheets. His test case was Brúarjökull, a glacier on the northern margin of Vatnajökull, Iceland. It flows over a smooth bed for $20 \mathrm{~km}$, from a location where its thickness is $600 \mathrm{~m}$, to a zero thickness margin. This glacier is entirely grounded. He showed that a good fit to measured surface elevations can be made using his model.

He initially states an equation for the ice-sheet surface elevation which has both vertical-plane shear and longitudinal 
stresses within the ice. However, he says this equation 'is quite tedious and very difficult to handle especially because of the [shear term]. It is therefore fortunate that [the shear] term appears to be small compared to the [basal sliding] term.' Then he drops the shear term and writes an equation in which driving stress is balanced by sliding resistance. He solves and analyzes this plug-flow model, for which we now state his equations in detail.

As is perhaps best known about Böðvarsson's work, he chooses the surface mass-balance rate to be

$$
M=a\left(H-H_{\text {ela }}\right),
$$

for a mass-balance gradient $a>0$ and equilibrium-line altitude $H_{\text {ela }}$. Böðvarsson's basal resistance formula scales with the overburden pressure, so that the basal shear stress is

$$
\tau_{\mathrm{b}}=-\beta u=-k \rho g H u \text {. }
$$

He then writes the ice flux as $u H=-(H / k) H_{x}$ or equivalently the ice velocity as

$$
u=-\frac{1}{k} H_{x} .
$$

(We return to Eqn (11) below, explaining it in modern language.) Combining Eqns (9) and (11) with mass continuity (Eqn (1)) yields eqn (17) of Böðvarsson (1955), namely

$$
a\left(H-H_{\text {ela }}\right)+\left(k^{-1} H H_{x}\right)_{x}=0 .
$$

His thickness solution to this equation is (Böðvarsson, 1955, eqns (18) and (23))

$$
H(x)=H_{0}\left[1-\left(x / L_{0}\right)^{2}\right],
$$

where $H_{0}=1.5 H_{\text {ela }}$ and $a k L_{0}^{2}=9 H_{\text {ela }}$ (Böðvarsson, 1955, eqn (24)).

Despite its simplicity, Eqn (13) is an exact solution to Eqn (12), with boundary condition $H(0)=H_{0}$, as the reader may verify. The formula of Eqn (13) defines the solid parabola shown in Figure 1. Böðvarsson does not offer a reason why there should be such a simple quadratic solution to Eqn (12). His solution seems to be a new result for a narrow class of nonlinear second-order ordinary differential equations (ODEs) like Eqn (12) (Appendix A). Seeking a polynomial solution to Eqn (12) with the single boundary condition $H(0)=H_{0}$, as in Appendix A, generates a location, $x=L_{0}$, where both the flux and the thickness are zero. Böðvarsson explicitly considers the solution, Eqn (13), as solving a free-boundary problem in this sense.

Böðvarsson had a particular 'sliding law' in mind, namely Eqn (10), in which the sliding coefficient scales with overburden pressure. The modern reader may protest that $\beta$ should instead be a function of (e.g. proportional to) effective pressure, $N=\rho g H-P$, where $P$ is the subglacial water pressure. However, it is not unreasonable to suppose that $P$ also scales with (is roughly a fixed fraction, $\lambda$, of) overburden pressure. In that case we have equations $\beta=c N$, $N=\rho g H-P$ and $P=\lambda \rho g H$, which combine to give $\beta=c(1-\lambda) \rho g H$. Thus, if the reader so wishes, in Böðvarsson's model we can write $\beta=k \rho g H$ with $k=c(1-\lambda)$.

Böðvarsson's 1955 paper was apparently first cited by Weertman (1961), who decided that his sliding, plug-flow physics should be replaced by a shear deformation model more like the shallow-ice approximation. This replacement seems to have influenced readers from then on. The surface balance parameterization, Eqn (9), also reappears in Weertman (1961), among other places. It parameterizes a potential climatic instability, which was Böðvarsson's,
Weertman's and most readers', major interest. We are, however, interested now in Böðvarsson's solution to the iceflow equations themselves.

\section{An SSA re-interpretation}

We have claimed that Eqn (13) exactly solves a combination of the steady flowline mass-continuity equation (Eqn (1)) and the SSA stress-balance equation (Eqn (2)), but the source of Eqn (11) may be less clear. However, suppose we look for solutions of Eqns (1) and (2), with constant vertically integrated longitudinal stress, $T_{X}=0$. In that case Eqn (2) and the scaling, Eqn (10), implies Böðvarsson's formula for the velocity, namely Eqn (11). Furthermore, Eqns (1) and (9) then give Böðvarsson's main equation, Eqn (12). That is, if (i) $T_{x}=0$, (ii) sliding resistance is linear and scales with overburden pressure and (iii) climatic-basal mass-balance rate is proportional to elevation above the equilibrium line, then we can recover a simple parabolic profile for $H(x)$, namely Eqn (13), which is a solution to Eqn (12).

But what does the condition $T_{x}=0$ imply as a relation among the modeled quantities? Given a thickness profile $H(x)$ and a strain-rate profile $u_{x}(x)$, we may interpret $T_{x}=0$ as a statement of variable ice hardness, $B(x)$. Generally, variation in hardness can be physically explained (e.g. in numerical models that use the SSA in a temperaturedependent way (Bueler and Brown, 2009)), so assuming that hardness is $x$-dependent requires no conceptual extensions in the marine ice-sheet modeling context. Numerical models can therefore use the exact solution we are building for verification without requiring unusual modifications.

Thus the variable hardness, $B(x)$, used here allows us to 'manufacture' an exact solution (Bueler and others, 2005). Specifically, the assumption $T=T_{0}$ in Eqn (3) yields a formula for ice hardness in grounded ice:

$$
B(x)=\frac{T_{0}}{2 H\left|u_{x}\right|^{(1 / n)-1} u_{x}} .
$$

The value $T_{0}$ in Eqn (14) will be set by a downstream stress condition, just as it is in many models for flowline ice shelves (e.g. Schoof, 2007; Pattyn and others, 2012).

\section{Extending the exact solution to floating ice}

Two well-known observations are relevant to the argument in the next paragraphs: (i) For floating ice with $\beta=0$, Eqns (1-7) also have a known exact solution, specifically in the case where the climatic-basal mass-balance rate, $M$, and the ice hardness, B, are constant (Van der Veen, 1983, 2013); (ii) The vertically integrated longitudinal stress, $T$, in a flowline ice shelf (i.e. one without lateral stresses) satisfies Eqn (7) at each location, $x$, in the shelf, i.e. $T(x)=$ $\frac{1}{2} \omega \rho g H(x)^{2}$, because this is a first integral of Eqn (2) if $\beta=0$.

Based on these observations we can construct a marine ice-sheet exact solution by connecting Böðvarsson's grounded solution to floating ice. Note the grounding-line thickness is $H\left(x_{\mathrm{g}}\right)=\left(\rho_{\mathrm{w}} / \rho\right) z_{\mathrm{o}}$ if we take $b=0$. Then from Böðvarsson's thickness solution (Eqn (13)) we can determine $x_{\mathrm{g}}$. At $x_{\mathrm{g}}$, from Eqns (11) and (13), we also know $u\left(x_{\mathrm{g}}\right)$. For $x_{\mathrm{g}} \leq x \leq x_{\mathrm{C}}$, the floating ice shelf, we then set $M(x)=M\left(x_{\mathrm{g}}\right)$ as constant from Eqn (9), thus making $M(x)$ continuous across the grounding line, while at the same time allowing us to use Van der Veen's construction, which is based on a constant climatic-basal mass-balance rate. The equation $T(x)=\frac{1}{2} \omega \rho g H(x)^{2}$ determines $T_{0}=T\left(x_{\mathrm{g}}\right)$ for use 


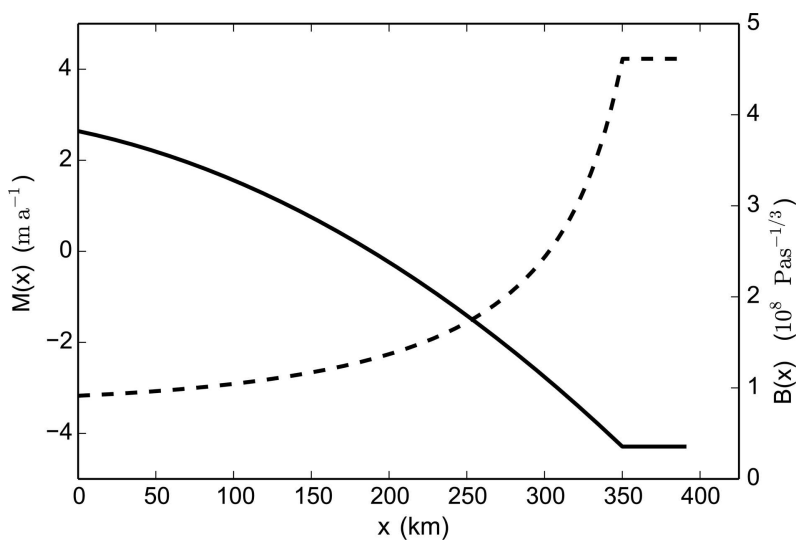

Fig. 3. The climatic-basal mass-balance rate, $M(x)$ (solid curve), and ice hardness, $B(x)$ (dashed curve), for the exact solution.

in Eqn (14), which determines $B\left(x_{\mathrm{g}}\right)$ in particular, and so we then set $B(x)=B\left(x_{\mathrm{g}}\right)$ for $x_{\mathrm{g}} \leq x \leq x_{\mathrm{C}}$, a constant, also needed in Van der Veen's construction.

The results of the above choices are the following formulas for an exact marine ice sheet satisfying our steady model equations (Eqns (1-8)). The velocity combines the Böðvarsson (1955) and Van der Veen (1983) results,

$$
u(x)= \begin{cases}\frac{2 H_{0}}{k L_{0}^{2}}\left(x+x_{\mathrm{a}}\right), & 0 \leq x \leq x_{\mathrm{g}} \\ u_{\mathrm{s}}(x), & x_{\mathrm{g}} \leq x \leq x_{\mathrm{C}}\end{cases}
$$

where the shelf velocity $u_{\mathrm{s}}(x)$ is defined by

$$
u_{\mathrm{s}}(x)^{n+1}=u\left(x_{\mathrm{g}}\right)^{n+1}+\frac{C_{\mathrm{s}}}{M\left(x_{\mathrm{g}}\right)}\left(Q_{\mathrm{s}}(x)^{n+1}-Q_{\mathrm{g}}^{n+1}\right),
$$

$C_{\mathrm{s}}=\left[\rho g \omega /\left(4 B\left(x_{\mathrm{g}}\right)\right)\right]^{n}, \quad Q_{\mathrm{g}}=u\left(x_{\mathrm{g}}\right) H\left(x_{\mathrm{g}}\right)$ and $Q_{\mathrm{s}}(x)=Q_{\mathrm{g}}+$ $M\left(x_{\mathrm{g}}\right)\left(x-x_{\mathrm{g}}\right)$. Similarly the thickness is

$$
H(x)= \begin{cases}H_{0}\left[1-\left(\frac{x+x_{\mathrm{a}}}{L_{0}}\right)^{2}\right], & 0 \leq x \leq x_{\mathrm{g}} \\ Q_{\mathrm{s}}(x) / u_{\mathrm{s}}(x), & x_{\mathrm{g}} \leq x \leq x_{\mathrm{c}} .\end{cases}
$$

Equations (15) and (17) define the continuous functions shown in Figure 2, using the specific values given in Table 2.

As described above, from the thickness, $H(x)$, and the velocity, $u(x)$, we can find continuous functions, $M(x)$ and $B(x)$, for the full flowline, using Eqns (9) and (14). These

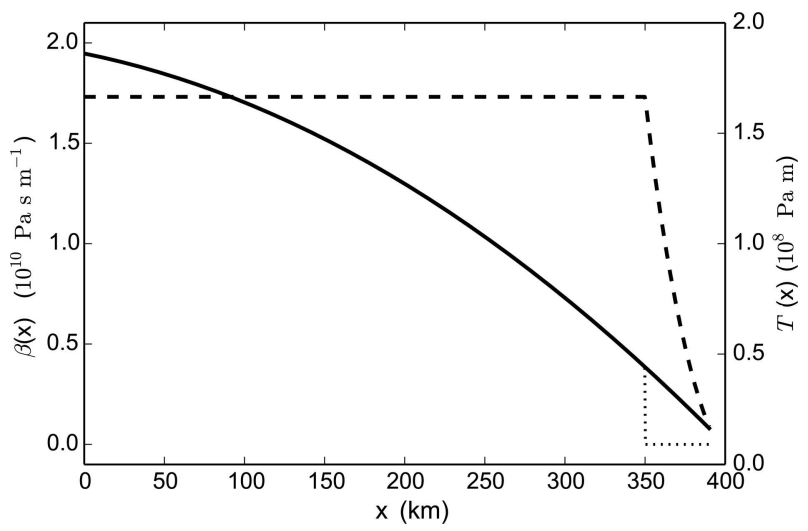

Fig. 4. The sliding coefficient, $\beta(x)$ (solid curve), and the vertically integrated longitudinal stress, $T(x)$ (dashed curve), for the exact solution. The solid curve shows $\beta=k \rho g H$ on both sides of the grounding line. The actual basal resistance experienced by the shelf drops to zero at the grounding line (dotted line).
Table 2. Specific values used in (above divider) or determined by

\begin{tabular}{|c|c|c|}
\hline Symbol & Description & Value \\
\hline$a$ & Surface mass-balance gradient & $0.003 a^{-1}$ \\
\hline$b$ & Bedrock elevation & $0 \mathrm{~m}$ \\
\hline$H_{0}$ & Thickness used in Eqn (13) & $3000 \mathrm{~m}$ \\
\hline$H_{\text {ela }}$ & Equilibrium-line altitude & $2000 \mathrm{~m}$ \\
\hline$k$ & Scaled sliding coefficient & $757.366 \mathrm{~s} \mathrm{~m}^{-1}$ \\
\hline$L_{0}$ & Length used in Eqn (13) & $500 \mathrm{~km}$ \\
\hline$x_{\mathrm{a}}$ & Offset & $100 \mathrm{~km}$ \\
\hline$z_{\mathrm{O}}$ & Ocean surface elevation & $504.572 \mathrm{~m}$ \\
\hline$H(0)$ & Thickness at $x=0$ & $2880 \mathrm{~m}$ \\
\hline$u(0)$ & Ice velocity at $x=0$ & $100 \mathrm{ma}^{-1}$ \\
\hline$x_{\mathrm{g}}$ & Location of g.l. & $350 \mathrm{~km}$ \\
\hline$B\left(x_{\mathrm{g}}\right)$ & Ice hardness at g.I. & $4.614 \times 10^{8} \mathrm{Pas} \mathrm{s}^{1 / 3}$ \\
\hline$H\left(x_{g}\right)$ & Thickness at g.l. & $570 \mathrm{~m}$ \\
\hline$M\left(x_{\mathrm{g}}\right)$ & Mass-balance rate at g.l. & $-4.290 \mathrm{ma}^{-1}$ \\
\hline$T\left(x_{\mathrm{g}}\right)$ & Stress at g.l. & $1.665 \times 10^{8} \mathrm{~Pa} \mathrm{~m}$ \\
\hline$u\left(x_{\mathrm{g}}\right)$ & Ice velocity at g.l. & $450 \mathrm{~m} \mathrm{a}^{-1}$ \\
\hline$x_{\mathrm{C}}$ & Location of c.f. & $390 \mathrm{~km}$ \\
\hline$H\left(x_{\mathrm{C}}\right)$ & Thickness at c.f. & $182.938 \mathrm{~m}$ \\
\hline$T\left(x_{\mathrm{C}}\right)$ & Stress at c.f. & $0.171 \times 10^{8} \mathrm{~Pa} \mathrm{~m}$ \\
\hline$u\left(x_{\mathrm{C}}\right)$ & Ice velocity at c.f. & $464.092 \mathrm{ma}^{-1}$ \\
\hline
\end{tabular}
(below divider) the equations that define the exact solution shown in Figures 2-5. Note 'g.l.' = grounding line and 'c.f.' = calving front

functions are shown in Figure 3. Then we can use Eqn (3) to find $T(x)$; this is shown in Figure 4. In Figure 4 we also show the sliding coefficient, $\beta(x)$, which drops to zero discontinuously at $x_{\mathrm{g}}$. Finally Figure 5 shows a detail of the grounding line and floating ice in the exact solution.

The floating ice shelf is a relatively short $40 \mathrm{~km}$ (Figs 2 and 5). To explain, note that the equilibrium-line altitude, $H_{\text {ela }}$, in Böðvarsson's (1955) solution is high on the ice sheet because of its relation to the upstream ice thickness in the construction of the exact solution (i.e. $\left.H_{\text {ela }}=(2 / 3) H_{0}\right)$. This, in turn, implies $M\left(x_{\mathrm{g}}\right)$ is quite negative (Eqn (9); Fig. 3). Because Van der Veen's (1983) solution uses a constant climatic-basal mass-balance rate, and because we want continuity for $M(x)$, we therefore have an ice shelf experiencing rapid melting. The location of the calving front, $x_{c}$, must, of course, be put upstream of the location where the ice has melted away. As a result of these same factors we also see a rapid decline in the stress, $T(x)$, from its

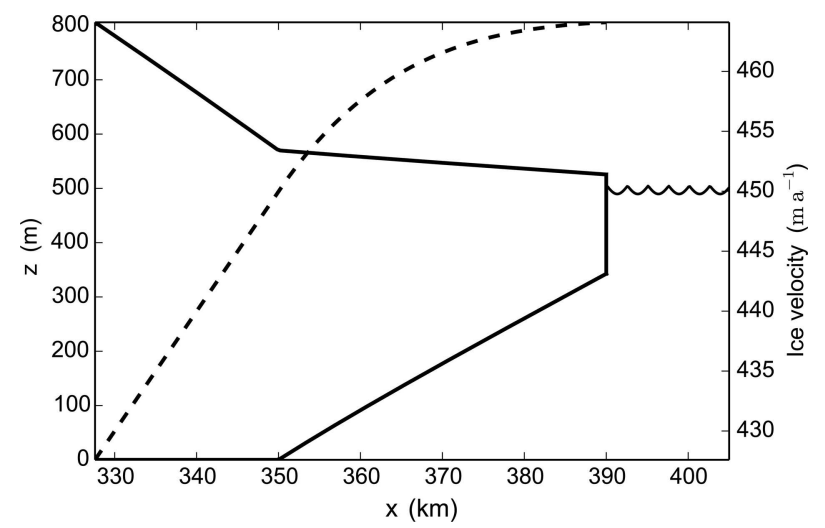

Fig. 5. Detail of Figure 2, showing the floating ice-shelf geometry and velocity. 

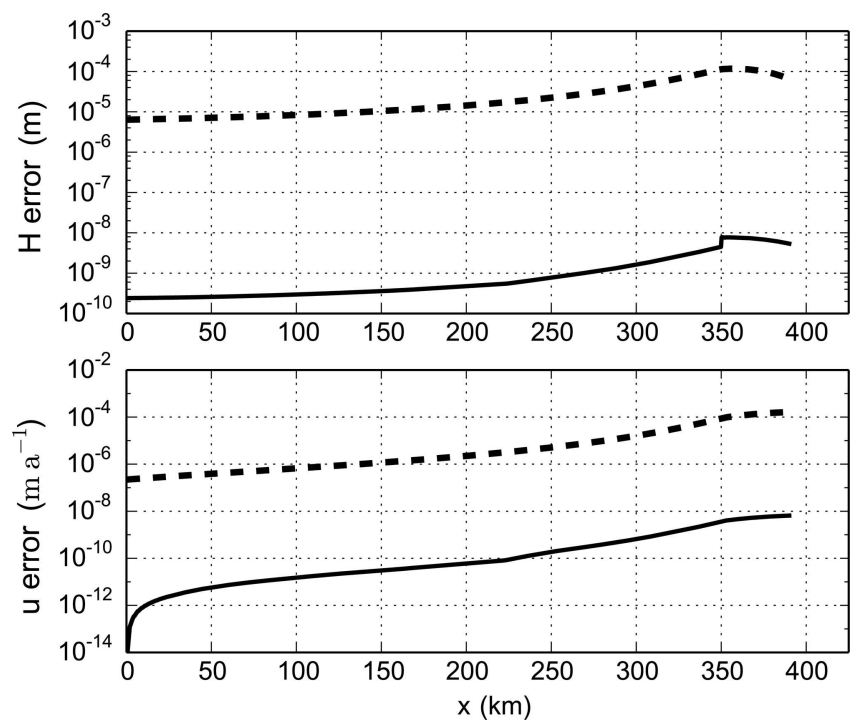

Fig. 6. Pointwise error in thickness (upper panel) and in velocity (lower panel) from an adaptive numerical ODE scheme. Both the 'cheating' case (solid curve), where we use the exactly correct initial value for $T$, and the 'realistic' case (dashed curve), where the shooting method converges on the correct initial value for $T$ by the bisection method, are shown.

constant grounded value to its small value at $x_{\mathrm{C}}$ (Fig. 4; Table 2). Though the ice shelf shown here is very wedgelike, the thickness, $H(x)$, for floating ice comes from Eqn (17), and it is not a linear function because $u_{\mathrm{s}}(x)$ is not constant on the shelf.

\section{NUMERICAL RESULTS \\ Verification of a grid-free 'shooting' numerical method}

In our steady flowline case the model equations form a twopoint boundary-value problem (BVP) for ODEs. Specifically, the three first-order ODEs, Eqns (1-3), are subject to two boundary conditions: Eqn (6) at $x=0$ and the calving-front stress condition, Eqn (7), at $x=x_{\mathrm{C}}$.

A nonlinear 'shooting' method (Press and others, 1992, section 17.1) applies to this problem. We use the correct values for $u(0)$ and $H(0)$ from Eqn (6) and guess an additional value, $T_{0}$, for $T(0)$. Then we use a numerical ODE initial-value problem (IVP) solver to compute a solution, $(\widetilde{u}(x), \widetilde{H}(x), \widetilde{T}(x))$, from $x=0$ to $x=x_{\mathrm{c}}$. The failure of this ODE IVP solution to satisfy boundary condition Eqn (7) is a measure of the wrongness of $T_{0}$. In fact, based on Eqn (7) we define the function

$$
F\left(T_{0}\right)=\widetilde{T}\left(x_{\mathrm{c}}\right)-\frac{1}{2} \omega \rho g \widetilde{H}\left(x_{\mathrm{c}}\right)^{2},
$$

and then we can apply a numerical method to find the solution (root), $\widehat{T}_{0}$, to the problem $F\left(T_{0}\right)=0$. This root gives us complete initial conditions, so that the ODE IVP solution also solves the two-point BVP, Eqns (1-7).

A robust root-finding method is bisection (Press and others, 1992, section 9.1). It is guaranteed to converge if $F$ is continuous and if an initial bracket is given, which is easy to find in this case. Regarding faster root-finding methods than bisection, such as Newton's method, we observe that $F^{\prime}$ may not exist because of the low regularity of the solution at the

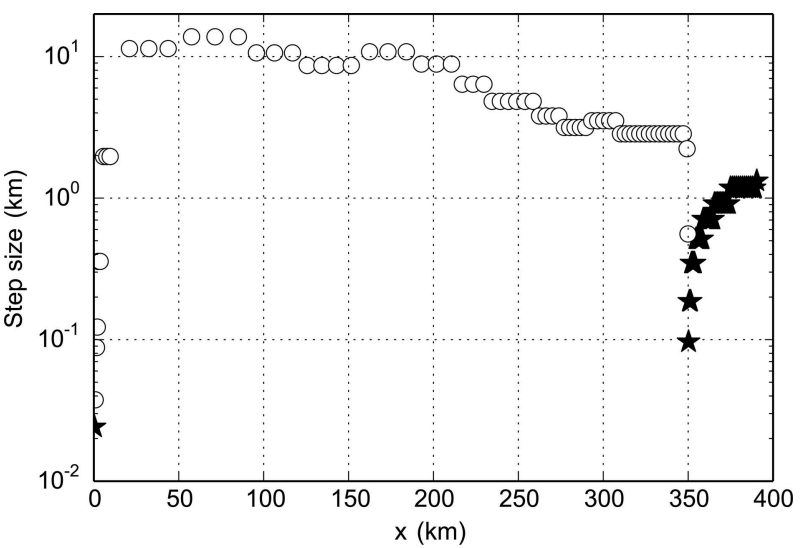

Fig. 7. The adaptive numerical ODE scheme in the 'realistic' case makes steps of $1-10 \mathrm{~km}$ in grounded ice, but at the grounding line, $x_{\mathrm{g}}=350 \mathrm{~km}$, the step size is reduced to a few hundred meters. The adaptive mechanism automatically switches from a stiff method where grounded (circles), to a non-stiff method where floating (stars).

interior point, $x=x_{\mathrm{g}}$. However, by using our exact solution we will see clear evidence that the bisection iteration succeeds in finding the root, $\widehat{T}_{0}$, to many digits, despite the uncertain smoothness of $F$.

This 'shooting' method has the advantage that the advanced step-size control mechanism of an ODE IVP solver determines the spatial gridpoints, so as to solve the ODEs to a desired tolerance. Thereby we avoid an a priori choice of the grid, and in this sense the method is grid-free. In this case we use LSODA from the ODEPACK collection (Hindmarsh, 1983), because it both automatically adjusts step-size to achieve desired tolerance and automatically switches method when stiffness (Press and others, 1992, section 16.6) is detected.

We now apply this grid-free procedure to the same problem for which we have the exact solution (given in Eqns (15-17)). Using relative tolerance $10^{-12}$ and absolute tolerance $10^{-14}$ for LSODA we get the numerical error shown in Figure 6. We have shown the error in two runs, one in which we have used the exactly correct initial value, $T_{0}$, ('cheating') and one in which we start with a large initial bracket on $T_{0}$ and converge on the correct calving-front boundary condition through shooting and bisection ('realistic'). In the 'cheating' runs we see that the numerical error just from solving the ODE, i.e. independent of errors in boundary conditions, is quite small, in the 10th or 11th digit for $H$ and $u$. The much larger error seen in the 'realistic' case suggests, however, that $F$ in Eqn (18) is significantly irregular. Apparently, matching the calving-front boundary condition by numerical shooting from upstream causes the loss of 4 or 5 digits of accuracy. Nonetheless, even in this 'realistic' case our numerical method achieves 6- or 7-digit accuracy over the whole domain, including in the immediate vicinity of the grounding line. Note that though the peak thickness error is near the grounding line, it is only modestly larger than errors elsewhere.

The ODE solver also detects the grounding line as a point of transition to shorter (spatial) steps, as seen in Figure 7. More significantly, however, the grounded ice requires a stiff method while the floating ice allows a non-stiff one, according to the automatic switch mechanism in LSODA. Note that high accuracy (e.g. 6 or 7 digits) is achieved in the 


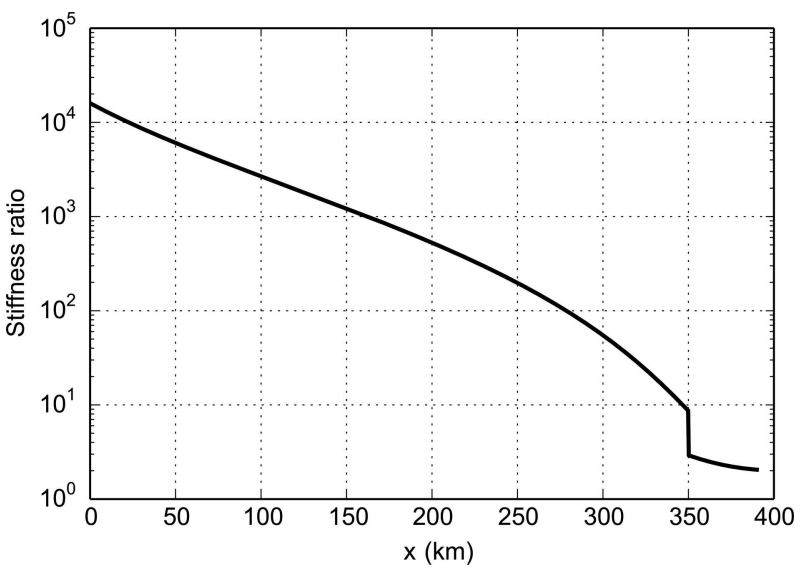

Fig. 8. Stiffness ratio $\left|\operatorname{Re}\left(\lambda_{1}\right)\right| /\left|\operatorname{Re}\left(\lambda_{3}\right)\right|$ for the linearized problem, Eqn (21), where $\lambda_{i}$ are the eigenvalues of $A(x)$ in Eqn (21).

'realistic' case, despite rather large grid spacing in the grounded ice, with large portions at $5-10 \mathrm{~km}$ spacing. The spacing drops to a minimum of $100 \mathrm{~m}$ just downstream of the grounding line at $x_{\mathrm{g}}=350 \mathrm{~km}$.

In all numerical tests we have treated $M(x)$ as a predetermined field (i.e. the one shown in Fig. 3). This removes the climatically important elevation-accumulation feedback, and the associated instability, of interest to Böðvarsson (1955) and others. This feedback can, however, be restored by using Eqn (9) to determine $M$ from $H$.

\section{Linearization around the exact solution}

The above numerical evidence shows that a distinct change in stiffness occurs at the grounding line. To analyze this we linearize the model equations around the exact solution. Denote the exact solution $\left(\widehat{u}_{0}, \widehat{H}_{0}, \widehat{T}_{0}\right)$ and consider a small perturbation $u=\widehat{u}+\epsilon \widetilde{u}, \quad H=\widehat{H}+\epsilon \widetilde{H}$ and $T=\widehat{T}+\epsilon \widetilde{T}$. Denote the column vector of perturbations by $\mathbf{w}=[\widetilde{u}, \widetilde{H}, \widetilde{T}]^{\top}$. Assuming $\widehat{u}_{x}>0$, Eqns (1-3) imply that, to first order in $\epsilon$, the perturbation solves this linear ODE system in grounded ice:

$$
\begin{aligned}
& {\left[\begin{array}{ccc}
\frac{2}{n} B \widehat{H}\left(\widehat{u}_{x}\right)^{p} & 0 & 0 \\
\widehat{H} & \widehat{u} & 0 \\
0 & -\rho g \widehat{H} & 1
\end{array}\right] \mathbf{w}_{x}} \\
& \quad=\left[\begin{array}{ccc}
0 & -2 B\left(\widehat{u}_{x}\right)^{1 / n} & 1 \\
-\widehat{H}_{x} & -\widehat{u}_{x} & 0 \\
-k \rho g \widehat{H} & -k \rho g \widehat{u}+\rho g \widehat{H}_{x} & 0
\end{array}\right] \mathbf{w},
\end{aligned}
$$

where $p=\frac{1}{n}-1$. In floating ice, only the last rows differ from Eqn (19):

$$
\left[\begin{array}{ccc} 
& \cdots & \\
0 & -\omega \rho g \widehat{H} & 1
\end{array}\right] \mathbf{w}_{x}=\left[\begin{array}{ccc} 
& \cdots & \\
0 & \omega \rho g \widehat{H}_{x} & 0
\end{array}\right] \mathbf{w} .
$$

If we define $L(x)$ and $R(x)$ to be the left- and right-side matrices in Eqns (19) and (20) then w solves

$$
\mathbf{w}_{x}=A(x) \mathbf{w}
$$

along its whole length, where $A(x)=L(x)^{-1} R(x)$.

Linear ODE system Eqn (21) is stiff if there is a large ratio of magnitudes in the eigenvalues of $A(x)$ (Press and others, 1992). Because the entries and eigenvalues of $A(x)$ are exactly computable using the exact solution values,
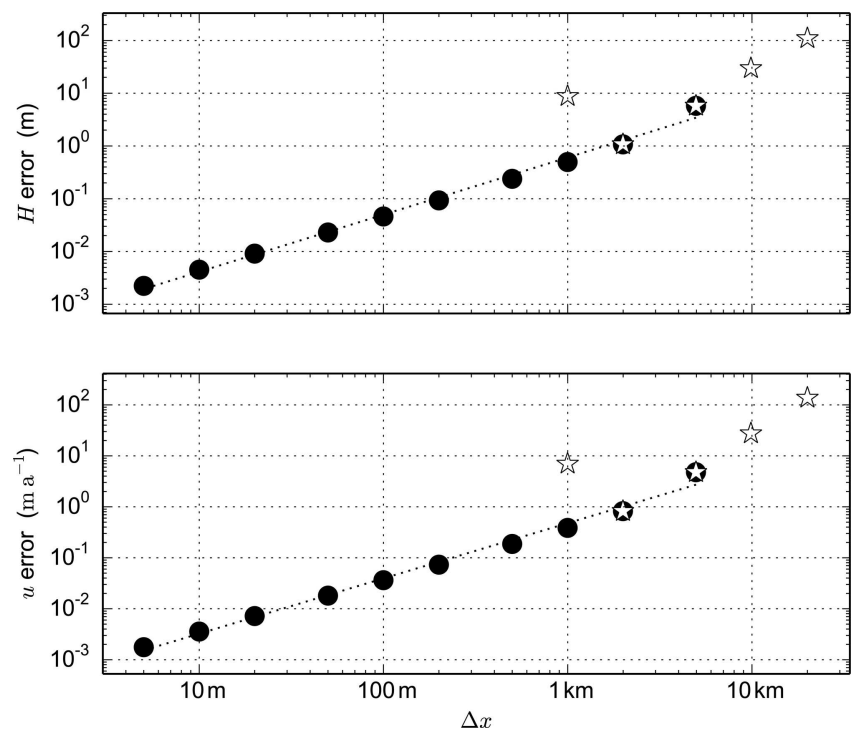

Fig. 9. Maximum errors in ice thickness (upper panel) and velocity (lower panel) on grids with spacing from $20 \mathrm{~km}$ to $5 \mathrm{~m}$. When initialized with the exact solution, the numerical scheme converges at a rate $\Delta x^{1.08}$ for both thickness and velocity (large dots plus dotted line). For a more realistic initial iterate the convergence rate is initially good, but at resolutions $<1 \mathrm{~km}$ the Newton iteration fails to converge (stars).

$(\widehat{u}(x), \widehat{H}(x), \widehat{T}(x))$, we can plot (Fig. 8) the $x$-dependent 'stiffness ratio' for $A(x)$, namely the ratio of absolute values of the real parts of the largest and smallest eigenvalues of $A(x)$, along the whole length of the flowline. Note this ratio is small in the non-stiff floating ice. This ratio is independent of the direction of integration (i.e. upstream versus downstream). The stiffness ratio is by no means the last word on quantifying stiffness, which turns out to be difficult generally (e.g. Higham and Trefethen, 1993).

We believe that the strong stiffness contrast at the grounding line is significant in explaining large neargrounding-line errors made by gridded numerical methods (Gladstone and others, 2010; Pattyn and others, 2012). The stiffness ratio drops by a factor of almost ten at the grounding line, though it is largest in the interior part of the grounded ice. It is possible that models of modified effective pressure near the grounding line (Leguy and others, 2014), in sliding laws that are parameterized by effective pressure (Schoof, 2005), can smooth this stiffness contrast.

\section{Verification of a fixed-grid finite-difference numerical method}

We also implemented an equally spaced, second-order, finite-difference scheme using Newton iteration (Appendix B). Our exact solution allows us to measure, for the first time in a rapidly sliding marine ice-sheet context, the errors from such a numerical scheme of the common type implemented in practical marine ice-sheet models (e.g. Pollard and DeConto, 2009; Winkelmann and others, 2011).

Figure 9 shows that the maximum numerical thickness and velocity errors are observed to converge at a rate much slower than the optimal $\mathrm{O}\left(\Delta x^{2}\right)$ rate under grid refinement (Morton and Mayers, 2005). This is essentially because of the loss of smoothness in the exact solution at the grounding line. By contrast, use of the grounded-only Böðvarsson (1955) exact solution, without a grounding line, confirms 
that the same finite-difference method gives optimal $\mathrm{O}\left(\Delta x^{2}\right)$ convergence (not shown).

It is important to distinguish the errors attributable to the finite-difference discretization itself from errors attributable to imperfect convergence of the nonlinear iterative solver that is applied to solve the discretized equations. To evaluate the former type of errors we initialized the nonlinear solver with the exact solution values. These are not the exact solutions of the discretized equations but they are (obviously) close. We see converged solutions to the discretized equations down to $5 \mathrm{~m}$ grids. The resulting thickness and velocity errors, at the $\mathrm{mm}$ and $\mathrm{mm}^{-1}$ level, respectively (Fig. 9), are larger than from the adaptive (grid-free) higher-order ODE method above (cf. Fig. 6). Nonetheless errors at this level are certainly acceptable, if they were to represent the realistic case.

However, if we use a simple 'wedge' initial iterate, which has a linear thickness profile from the upstream initial condition, $H(0)$, down to $300 \mathrm{~m}$ at the calving front, and a similar linear velocity profile increasing from $u(0)$ to $300 \mathrm{~m} \mathrm{a}^{-1}$ at the calving front, then we see more realistic results, that reflect the experience of ice-sheet modelers addressing these equations. Here the initial iterate is relatively far from the exact solution. Difficulties arise in the global convergence behavior of the Newton solver, even though standard line-search techniques are used in these computations (Kelley, 1987). For grids finer than $1 \mathrm{~km}$ the iteration for this scheme does not converge, apparently because the Jacobian matrix is not providing useful directional information as to the location of the solution of the discretized equations.

Our results suggest a lack of nonlinear solver robustness that we attribute to the non-smooth, stiffness-contrasting properties of the problem near the grounding line. Grounding-line parameterizations (e.g. Gladstone and others, 2010; Feldmann and others, 2014) may act like homotopy continuation methods (Kelley, 1987) to improve global solver behavior in this case, but such considerations go beyond the scope of this paper. No regularization of grounding-line discontinuities and slope discontinuities in the formulas for $\beta(x)$ and $h(x)$ (respectively) were applied in this paper.

\section{CONCLUSION}

As noted by Bueler and others (2005), Wesseling (2001) and many others, verification of numerical methods is a valuable first step in effective numerical modeling of realistic flows. This is especially so in geophysical flows, where validation by comparison with controlled laboratory experiments is difficult. Thus, the rediscovery of an exact solution to a marine ice-sheet problem is a welcome development. Even though this solution is for a steady-state and flat-bed case, it provides a partial alternative to using hard-to-interpret intercomparison results (Pattyn and others, 2012).

Because this solution is found in some of the first work in theoretical glaciology (Böðvarsson, 1955), we have recovered an early approach to sliding dynamics. The rapid-sliding case turns out to be one of the first dynamical situations examined, even though other early efforts at global views of ice dynamics tended toward the plastic-ice (Orowan comment in BGS, 1949; Nye, 1952), frozen-bed (Vialov, 1958) and vertical-shear dominated (Weertman, 1961) models, and these came to dominate the field until recent decades.

Citations of Böðvarsson (1955) usually relate to its theory of climatic instability for glaciers and ice sheets, in which surface mass-balance rate depends on elevation. The only citation exception to this pattern known to the current author is that Fowler (1992) refers to the basal-sheardominated dynamics of Böðvarsson (1955) as 'approximate results'. We hope that the current work revives interest in Böðvarsson's ice-dynamical solution and corrects the misreading of his results as approximate.

Application of the new exact solution here, which includes a floating extension, also reveals one feature of the marine ice-sheet problem that we feel has been overlooked, namely that there is a strong stiffness contrast at the grounding line, in the sense of differential equations. This is, conceptually, in addition to the loss of smoothness seen at the grounding line. Both smoothness and stiffness must be addressed by numerical methods. Modelers should have more than grid refinement in mind as they attempt to model grounding lines correctly.

\section{ACKNOWLEDGEMENTS (AND ERRATUM)}

Comments from referees R. Gladstone and K. Hutter have focused and improved the paper. Thanks to G. Aðalgeirsdóttir, H. Blatter and H. Björnsson for tracking down a copy of Böðvarsson (1955) and a bibliography of the works of Gunnar Böðvarsson. Bueler and others (2005) incorrectly identify the constant accumulation SIA solution as 'Bodvarsson (1955)-Vialov (1958)'. In fact it is attributable only to Vialov. Finally, note that spellings of Böðvarsson include 'Bodvardsson' (e.g. in Böðvarsson, 1955) and 'Bodvarsson' in the literature.

\section{REFERENCES}

Balay S and 13 others (2014) PETSc users manual. (Tech. Rep. ANL95/11, Rev. 3.4) Argonne National Laboratory, Argonne, IL

Böðvarsson G (1955) On the flow of ice-sheets and glaciers. Jökull, 5, 1-8

British Glaciological Society (BGS) (1949) Joint meeting of the British Glaciological Society, the British Rheologists' Club and the Institute of Metals. J. Glaciol., 1(5), 231-240 (doi: 10.3189/ 002214349793702827)

Bueler E and Brown J (2009) Shallow shelf approximation as a 'sliding law' in a thermomechanically coupled ice sheet model. J. Geophys. Res., 114(F3), F03008 (doi: 10.1029/2008JF001179)

Bueler E, Lingle CS, Kallen-Brown JA, Covey DN and Bowman LN (2005) Exact solutions and verification of numerical models for isothermal ice sheets. J. Glaciol., 51(173), 291-306 (doi: 10.3189/172756505781829449)

Cogley JG and 10 others (2011) Glossary of glacier mass balance and related terms. (IHP-VII Technical Documents in Hydrology 86) UNESCO-International Hydrological Programme, Paris

Feldmann J, Albrecht T, Khroulev C, Pattyn F and Levermann A (2014) Resolution-dependent performance of grounding line motion in a shallow model compared with a full-Stokes model according to the MISMIP3d intercomparison. J. Glaciol., $60(220)$, 353-360 (doi: 10.3189/2014JoG13J093)

Fowler AC (1992) Modelling ice sheet dynamics. Geophys. Astrophys. Fluid Dyn., 63(1-4), 29-65 (doi: 10.1080/ 03091929208228277)

Gladstone RM, Payne AJ and Cornford SL (2010) Parameterising the grounding line in flow-line ice sheet models. Cryosphere, 4(4), 605-619 (doi: 10.5194/tc-4-605-2010)

Higham DJ and Trefethen LN (1993) Stiffness of ODEs. BIT Num. Math., 33(2), 285-303 (doi: 10.1007/BF01989751)

Hindmarsh AC (1983) ODEPACK, a systematized collection of ODE solvers. In Stepleman RS, Carver M, Peskin R, Ames WF and Vichnevetsky $\mathrm{R}$ eds. Scientific computing, applications of 
mathematics and computing to the physical sciences. 10th IMACS World Congress on Systems Simulation and Scientific Computation, 8-13 August 1982, Montreal, Canada. (IMACS Transactions on Scientific Computation 1) North-Holland, Amsterdam, 55-64

Kelley CT (1987) Solving nonlinear equations with Newton's Method. Society for Industrial and Applied Mathematics, Philadelphia, PA

Leguy GR, Asay-Davis XS and Lipscomb WH (2014) Parameterization of basal hydrology near grounding lines in a onedimensional ice sheet model. Cryosphere, 8(4), 1239-1259 (doi: 10.5194/tc-8-1239-2014)

MacAyeal DR (1989) Large-scale ice flow over a viscous basal sediment: theory and application to Ice Stream B, Antarctica. J. Geophys. Res., 94(B4), 4071-4087 (doi: 10.1029/ JB094iB04p04071)

Morton KW and Mayers DF (2005) Numerical solution of partial differential equations: an introduction, 2nd edn. Cambridge University Press, Cambridge

Nye JF (1952) A method of calculating the thickness of ice sheets. Nature, 169(4300), 529-530

Pattyn F and 18 others (2012) Results of the Marine Ice Sheet Model Intercomparison Project, MISMIP. Cryosphere, 6(3), 573-588 (doi: 10.5194/tc-6-573-2012)

Pollard D and DeConto RM (2009) Modelling West Antarctic ice sheet growth and collapse through the past five million years. Nature, 458(7236), 329-332 (doi: 10.1038/nature07809)

Press WH, Teukolsky SA, Vetterling WT and Flannery BP (1992) Numerical recipes in C: the art of scientific computing, 2nd edn. Cambridge University Press, Cambridge

Schoof C (2005) The effect of cavitation on glacier sliding. Proc. $R$. Soc. London, Ser. A, 461(2055), 609-627 (doi: 10.1098/rspa. 2004.1350)

Schoof C (2006) A variational approach to ice stream flow. J. Fluid Mech., 556, 227-251 (doi: 10.1017/S0022112006009591)

Schoof C (2007) Marine ice-sheet dynamics. Part 1. The case of rapid sliding. J. Fluid Mech., 573, 27-55 (doi: 10.1017/ S0022112006003570)

Van der Veen CJ (1983) A note on the equilibrium profile of a free floating ice shelf. (IMOU Report V83-15) Rijksuniversiteit Instituut voor Meteorologie en Oceanografie, Utrecht

Van der Veen CJ (2013) Fundamentals of glacier dynamics, 2nd edn. CRC Press, Boca Raton, FL

Vialov SS (1958) Regularities of glacial shields movement and the theory of plastic viscous flow. IASH Publ. 47 (Symposium at Chamonix, 1958 - Physics of the Movement of the Ice), 266-275

Weertman J (1961) Stability of ice-age ice sheets. J. Geophys. Res., 66(11), 3783-3792 (doi: 10.1029/JZ066i011p03783)

Weis M, Greve R and Hutter K (1999) Theory of shallow ice shelves. Contin. Mech. Thermodyn., 11(1), 15-50 (doi: 10.1007/s001610050102)

Wesseling P (2001) Principles of computational fluid dynamics. Springer Verlag, Berlin

Winkelmann R and 6 others (2011) The Potsdam Parallel Ice Sheet Model (PISM-PIK) - Part 1: Model description. Cryosphere, 5(3), 715-726 (doi: 10.5194/tc-5-715-2011)

\section{APPENDIX A: BÖĐVARSSON'S LITTLE THEOREM}

Böðvarsson (1955) does not identify a source for the exact parabolic thickness solution to his flow equations, and we have not been able to attribute it to earlier work. We summarize his result as the theorem that there is a unique polynomial solution, $y(x)$, to the nonlinear second-order differential equation

$$
\left(y y^{\prime}\right)^{\prime}=c_{1} y+c_{0}
$$

satisfying the single boundary condition, $y(0)=y_{0}>0$, subject to both an initial downslope assumption $\left(y^{\prime}(0) \leq 0\right)$ and to the technical inequality $2 c_{1} y_{0}+3 c_{0} \geq 0$.

Equation (A1) is eqn (17) of Böðvarsson (1955). The additional assumptions (i.e. initial downslope plus the technical inequality) are unstated, though he comments that there is 'one and only one solution which is admittable from the physical point of view'.

Here we justify this little theorem and, generally following Böðvarsson (1955), derive relations among parameters which allow a solution. The unique polynomial solution may be interpreted as solving a free-boundary problem for the first positive zero $x_{0}>0$ of $y(x)$. In Böðvarsson's context $x_{0}=L_{0}$ is the length of the glacier, the location of the margin in the everywhere-grounded case.

It is easy to see by substitution into Eqn (A1) that nontrivial solutions of degree $d$, i.e. of the form $y(x)=C x^{d}+($ lower degree) with $C \neq 0$, exist only if $d=2$. In that case we seek solutions that satisfy the boundary conditions $y(0)=y_{0}$ and $y^{\prime}(0) \leq 0$, so

$$
y(x)=y_{0}\left(1-\alpha x+\gamma x^{2}\right)
$$

for some $\alpha \geq 0$ and $\gamma$, which are to be determined (this is eqn (18) of Böðvarsson, 1955). Substitution of Eqn (A2) into Eqn (A1) gives the two equations

$$
3 y_{0}^{2} \alpha^{2}=2 c_{1} y_{0}+3 c_{0} \text { and } 6 y_{0} \gamma=c_{1} .
$$

These two relations determine $\alpha$ and $\gamma$ from $c_{0}$ and $c_{1}$. The first relation explains the technical inequality, noting $3 y_{0}^{2} \alpha^{2} \geq 0$, of course.

In his main text, Böðvarsson (1955) relates four numbers to the unknown glacier thickness, which is $y(x)$ in the above formulas: the initial (upstream) ice thickness, $H_{0}$, an ablation gradient, $a>0$, the equilibrium-line altitude, $H_{\text {ela, }}$ and a scaled sliding coefficient, $k>0$. He has $c_{0}=k a H_{\text {ela }}$ and $c_{1}=-k a$ in Eqn (A1), so the technical inequality says $3 H_{\text {ela }} \geq 2 H_{0}$ after simplification. This causes the equilibrium-line altitude to be relatively high on the glacier.

\section{APPENDIX B: A FINITE-DIFFERENCE SCHEME}

The steady-state equations for mass continuity (Eqn (1)) and stress balance (Eqn (2)) form a coupled system that can be approximately solved by the centered, second-order finitedifference scheme described here. There is no claim that this scheme is optimal, but merely that it is a reasonable fixedgrid method for initial evaluation. Because we use it to solve a steady-state problem, it generalizes to the time-dependent case as an implicit method.

We define an equally spaced grid on the domain $\left[0, x_{\mathrm{C}}\right]$. Because the boundary condition at the calving front evaluates the stress, $T$, we put the right endpoint, $x_{C}$, at a 'staggered' location halfway between gridpoints. Thus if $N$ is the number of spaces we define $\Delta x=x_{c} /(N+1 / 2)$ and $x_{j}=j \Delta x$ for $j=0, \cdots, N+1$. Denote the numerical approximations $H_{i} \approx H\left(x_{i}\right)$ and $u_{i} \approx u\left(x_{i}\right)$. Let $x_{j}^{*}=x_{j}+\Delta x / 2$ be the staggered location for $j=0, \cdots, N$. Note $x_{\mathrm{C}}=x_{N}^{*}<x_{N+1}$. Denote $B_{j}^{*}=B\left(x_{j}^{*}\right)$ and $M_{j}^{*}=M\left(x_{j}^{*}\right)$.

The mass-continuity equation (Eqn (1)) is approximated by a second-order difference centered at the staggered location. For $j=0, \cdots, N$,

$$
\frac{u_{j+1} H_{j+1}-u_{j} H_{j}}{\Delta x}-M_{j}^{*}=0 .
$$

In Eqn (2) we avoid infinite viscosity by using a regularization 
(Schoof, 2006). Let $\epsilon=1 / x_{c}$ per year, i.e. a strain rate corresponding to $1 \mathrm{ma}^{-1}$ velocity change over the whole domain. Define

$$
F\left(u_{1}, u_{\mathrm{r}}\right)=\left[\left(\frac{u_{\mathrm{r}}-u_{\mathrm{l}}}{\Delta x}\right)^{2}+\epsilon^{2}\right]^{(1-n) / 2 n} \frac{u_{\mathrm{r}}-u_{\mathrm{l}}}{\Delta x},
$$

where $u_{\mid}$and $u_{\mathrm{r}}$ are velocity values to the left and right of a staggered gridpoint, respectively. Then we approximate the stress, $T$, at staggered points,

$$
T_{j}^{*}=B_{j}^{*}\left(H_{j}+H_{j+1}\right) F\left(u_{j}, u_{j+1}\right),
$$

for $j=0, \cdots, N$. Equation (2) is approximated by

$$
\frac{T_{j}^{*}-T_{j-1}^{*}}{\Delta x}-\beta_{j} u_{j}-\rho g H_{j} \frac{h_{j+1}-h_{j-1}}{2 \Delta x}=0
$$

for $j=1, \cdots, N$, where $\beta_{j}=k \rho g H_{j}$ if the ice is grounded at $x_{j}$ (i.e. if $\rho H_{j} \geq \rho_{\mathrm{w}}\left(z_{\mathrm{o}}-b\right)$ ) and $\beta_{j}=0$ if the ice is floating, and where $h_{j}=H_{j}+b$ if the ice is grounded and $h_{j}=\omega H_{j}+z_{\mathrm{o}}$ if the ice is floating. Thus Eqn (B4) applies, as stated, both for grounded and floating ice.

At this point we have $2 \mathrm{~N}+4$ scalar unknowns, namely $u_{j}$ and $H_{j}$ for $j=0, \cdots, N+1$. There are $2 N+1$ nonlinear equations in Eqns (B1) and (B4). The two upstream Dirichlet equations (Eqn (6)), namely $u_{0}=u(0)$ and $H_{0}=H(0)$, bring the number of equations to $2 \mathrm{~N}+3$. The following approximation of the calving-front condition (Eqn (7)), completes the system:

$$
\frac{1}{2} \omega \rho g\left(\frac{H_{N}+H_{N+1}}{2}\right)^{2}=T_{N^{\prime}}^{*}
$$

where $T_{N}^{*}$ is the approximation given in Eqn (B3).

Thus we have a system of $2 N+4$ nonlinear equations in the same number of unknowns. One can write this system abstractly as $\mathbf{F}(\mathbf{v})=0$. These equations are solved by Newton's method (Kelley, 1987), as implemented in the PETSc library (Balay and others, 2014). A residual evaluation function computes $\mathbf{F}(\mathbf{v})$ given $\mathbf{v}$. A finite-difference Jacobian matrix, $J=\mathbf{F}^{\prime}$, can then be computed by PETSc, and this allows us to solve systems up to size $N \approx 10^{3}$. We also implemented an exact Jacobian using by-hand differentiation of the above formulas. For initial guesses sufficiently near the exact solution, this exact Jacobian permits solutions of the system for up to $N=10^{5}$. A full analysis of the robustness and convergence rate of this Newton solver is beyond the scope of the current paper. 